\title{
Optical Measurements and Speckle Photography for Thermotropic Liquid Crystals Mixtures
}

\author{
Ayman A. Zaki \\ Physics Department, Faculty of Science, Benha University, Benha, Egypt \\ Email: ayman_a73@ hotmail.com
}

Received October 22, 2012; revised November 22, 2012; accepted December 2, 2012

Copyright (C) 2013 Ayman A. Zaki. This is an open access article distributed under the Creative Commons Attribution License, which permits unrestricted use, distribution, and reproduction in any medium, provided the original work is properly cited.

\begin{abstract}
In this work, an experimental approach of speckle photography was used for measuring birefringence of thermotropic liquid crystals mixtures at different wavelengths in the visible region. Also the dispersion relations were investigated. The values of the refractive indices were measured for these mixtures of thermotropic liquid crystals in isotropic and liquid crystal phase at different wavelengths. The effect of the end group of these LCs used on the values of birefringence was investigated and discussed.
\end{abstract}

Keywords: Thermotropic Liquid Crystals Mixtures; Interference; Birefringence; Speckle Photography

\section{Introduction}

Liquid crystal technology has a major effect on many fields of science and engineering, as well as devices technology. Applications for this special kind of material are still being discovered and continued to provide effective solutions to many different problems.

The complex optical properties of thin films of polymer dispersed liquid crystals, and their refractive indices, have been determined [1]. Birefringence measurements at the wavelength $0.6328 \mu \mathrm{m}$ for some common nematic liquid crystal cells were presented [2]. Refractive indices $\left(n_{e}, n_{o}\right)$ and birefringence $(\Delta n)$ for cholesteric and isotropic phases of cholesteryl carbonate, cholesteryl stearate at varying temperature were measured [3]. Transmittance spectra of a mica film and 4-cyano 49-pentyl biphenyl liquid crystal were acquired in the visible spectral region, and the dispersion curves of the refractive index and the birefringence were deduced [4]. New guidelines for selecting or synthesizing the liquid crystals with the desired birefringence were established [5]. The thermotropic liquid crystal (N-4-methoxybenzilidene-4butylaniline), entrapped on hydrogels, based on crosslinked polyacrylamide was studied [6]. The birefringence of the liquid crystal as functions of the temperature was measured with and without the power supply [7]. The voltage dependence of the birefringence, for a nematic liquid crystal mixture doped with two anthraquinone derivatives were predicted [8]. The temperature dependence of the two indices $n_{e}, n_{o}$ and of the optical activity for uniaxial liquid crystals was reported [9]. The processes of mesogen ordering in nematic and cholesteric sidechain polymer systems were studied [10]. Properties of anodic aluminum oxide film as a liquid crystal alignment material were studied [11].

This work deals with four thermotropic LCs compounds locally prepared and the structure of thermotropic liquid crystal materials used is 4-substituted phenyl-4alkoxy benzoates [12] which have the following structure:

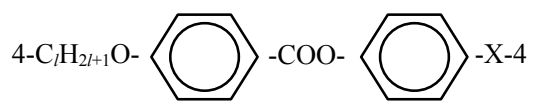

where $l$ is an integer number, and $\mathrm{X}$ is the extension group which is the electron-withdrawing $\mathrm{NO}_{2}$. In this work $l$ took the numbers of $6,8,14$, and 16 respectively. After that a mixture of liquid crystal of $l$ equal 6 and 14, and of $l$ equal 6,14 and 16 and other of 6, 8, 14 and 16 were used, where the composition per percentage mole was as in Table 1. Each material used is represented by the value of $l$ with a symbol "e" after each number referring to the end group $\mathrm{NO}_{2}$. Table 1 shows transition temperatures in Celsius degree for the compounds used in this work. The symbols C-A, C-N, A-N, A-I, and N-I mean the transition from, solid crystal to semictic A, solid crystal to nematic, semictic A to nematic, semictic A to isotropic and nematic to isotropic respectively. 
Table 1. Composition and transition temperatures $\left({ }^{\circ} \mathrm{C}\right.$ ) for compounds $\mathrm{C}_{l} \mathrm{H}_{2 l+1} \mathrm{O}-\mathrm{C}_{6} \mathrm{H}_{4}-\mathrm{COO}-\mathrm{C}_{6} \mathrm{H}_{4}-\mathrm{NO}_{2}$. Values between parentheses are those determined by the extrapolation method.

\begin{tabular}{cccccccccc}
\hline \multirow{2}{*}{ Mixture } & \multicolumn{3}{c}{ Composition $(\mathrm{mol} \%)$} & \multicolumn{5}{c}{ Transition Temp. $\left({ }^{\circ} \mathrm{C}\right)$} \\
\cline { 2 - 9 } & $l=6$ & $l=8$ & $l=14$ & $l=16$ & $\mathrm{~T}_{\mathrm{C}-\mathrm{A}}$ & $\mathrm{T}_{\mathrm{C}-\mathrm{N}}$ & $\mathrm{T}_{\mathrm{A}-\mathrm{A}}$ & $\mathrm{T}_{\mathrm{A}-\mathrm{I}}$ & $\mathrm{T}_{\mathrm{N}-\mathrm{I}}$ \\
\hline $6 \mathrm{e}$ & pure & - & - & - & - & - & - & - & $(71.2)$ \\
$8 \mathrm{e}$ & - & pure & - & - & 53.1 & - & 62.6 & - & 69.2 \\
$14 \mathrm{e}$ & - & - & pure & - & - & - & - & - & $(76.2)$ \\
$6 / 14 \mathrm{e}$ & 60.0 & - & 37.5 & - & 50 & - & 68.0 & - & - \\
$6 / 14 / 16 \mathrm{e}$ & 47.03 & - & 30.73 & 22.22 & 45.99 & - & - & 72.50 & - \\
$6 / 8 / 14 / 16 \mathrm{e}$ & 33.08 & 28.55 & 21.12 & 17.22 & - & 69.0 & - & - & $(78.0)$ \\
\hline
\end{tabular}

\section{Refractive Index Measurements}

Two different methods were used for measuring refractive indices of the materials used; one was Abbe refractometer equipped with hot water bath around the prisms with different wavelengths at different degrees of temperature. The temperature of water was controlled by a thermostat within $\pm 0.1{ }^{\circ} \mathrm{C}$. A white light source was used with interference filters of wavelengths $(640 \mathrm{~nm}, 577 \mathrm{~nm}, 546 \mathrm{~nm}$, $435.8 \mathrm{~nm})$, and a sodium lamp of wavelength $(589.3 \mathrm{~nm})$. The dispersion relations between refractive indices and wavelengths at certain temperature in isotropic and liquid crystal phase were shown in Figures 1-6, for (6e), (8e), (14e), (6/14e), (6/14/16e) and (6/8/14/16e).

Another method for measuring the values of refractive indices of the thermotropic LC materials used was by using the spectrophotometer device at different wavelengths of white light source. The value of refractive index was determined by measuring the reflectance values of material [13]. A slight difference in the accuracy of the values of refractive indices was noticed between $\approx 0.001$ to $\approx 0.01$ when comparing results between the measurements obtained from Abbe refractometer and the spectrophotometric technique.

\section{Birefringence Measurement}

Birefringence of the liquid crystals was measured by using speckle interferometry technique. The optical setup used is shown in Figure 7. The samples were put into an electric oven for measuring birefringence at a certain degrees of temperature in order to obtain the LC phase as shown in Table 1. The interference pattern for both ordinary and extraordinary components were magnified by $(q / p)$, where $p$ was the distance from the lens $\left(\mathrm{L}_{3}\right)$ to the diffuser (D), and $q$ is the distance from the lens $\left(\mathrm{L}_{3}\right)$ to the image $(\mathrm{H})$; $t$ was the thickness of the liquid crystal whose refractive index is $n$. The photographs were taken while the samples temperatures were heated and when they cooled. The setup of the optical arrangement in

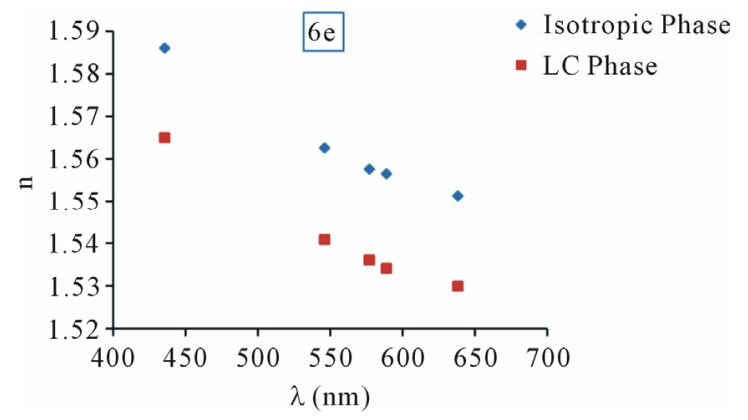

Figure 1. The relation between refractive index $(n)$ and wavelength $(\lambda)$ at certain temperature, $T=65^{\circ} \mathrm{C}$ in isotropic phase, and $55^{\circ} \mathrm{C}$ in $\mathrm{LC}$ phase for (6e) liquid crystal.

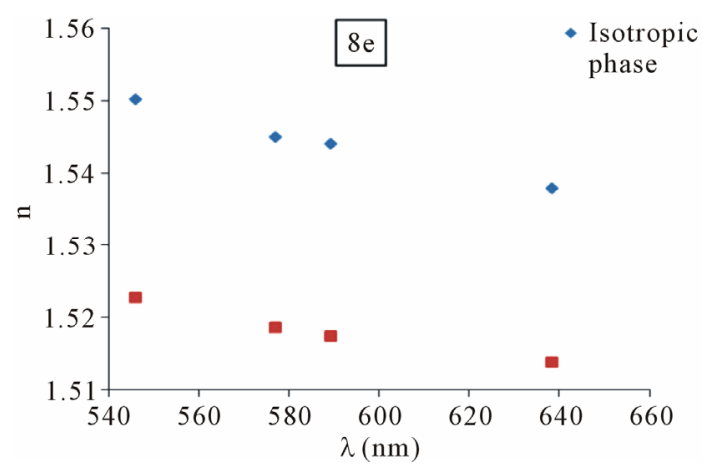

Figure 2. The relation between refractive index $(n)$ and wavelength $(\lambda)$ at certain temperature, $T=73^{\circ} \mathrm{C}$ in isotropic phase, and $66^{\circ} \mathrm{C}$ in $\mathrm{LC}$ phase for (8e) liquid crystal.

Fourier plane for observing the Young fringes from the specklegram was used [14]. Plate 1 shows the interfereence Young's fringes from the specklegram of $(6 \mathrm{e}, 14 \mathrm{e}$, $6 / 14 \mathrm{e}$ and $6 / 14 / 16 \mathrm{e}$ ) thermotropic liquid crystals at wavelength $632.8 \mathrm{~nm}$.

The relationship of the shift $D$ between ordinary and extraordinary images and the spacing of the Young's fringes $\Lambda$ is given by [15]:

$$
D=f \lambda\left(\frac{p}{q \Lambda}\right)
$$




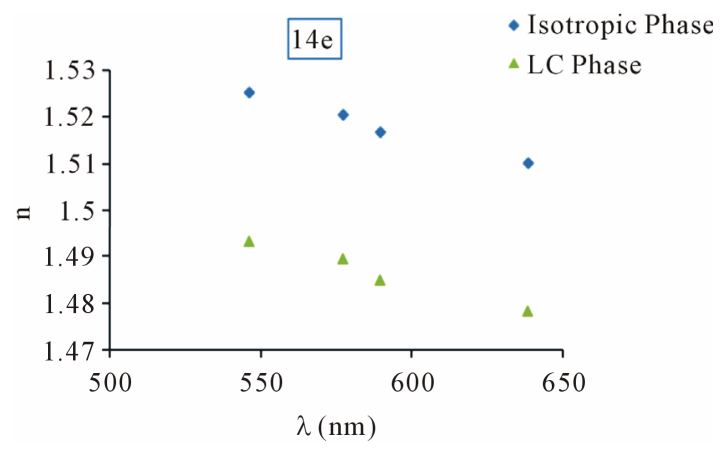

Figure 3. The relation between refractive index $(n)$ and wavelength $(\lambda)$ at certain temperature, $T=85^{\circ} \mathrm{C}$ in isotropic phase, and $75^{\circ} \mathrm{C}$ in LC phase for (14e) liquid crystal.

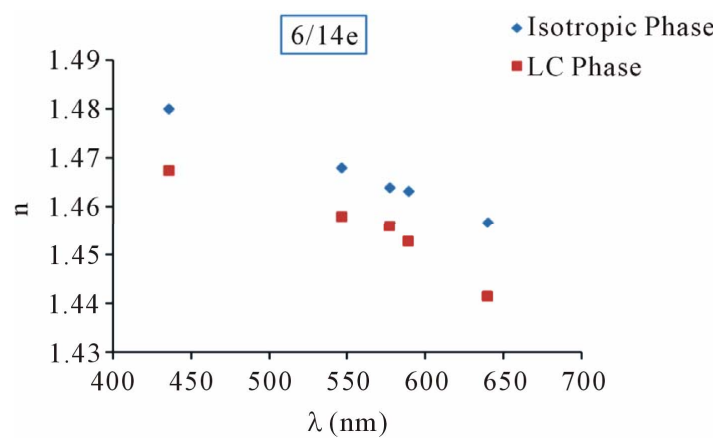

Figure 4. The relation between refractive index $(n)$ and wavelength $(\lambda)$ at certain temperature, $T=78^{\circ} \mathrm{C}$ in isotropic phase, and $70^{\circ} \mathrm{C}$ in $\mathrm{LC}$ phase for (6/14e) liquid crystal.

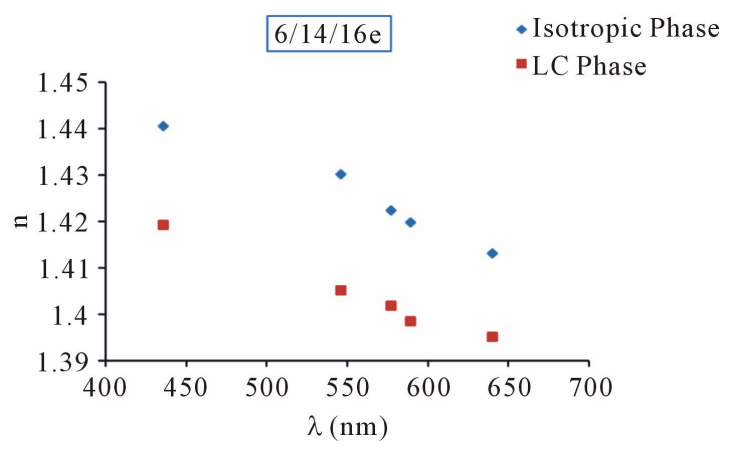

Figure 5. The relation between refractive index $(n)$ and wavelength $(\lambda)$ at certain temperature, $T=78^{\circ} \mathrm{C}$ in isotropic phase, and $65^{\circ} \mathrm{C}$ in LC phase for $(6 / 14 / 16 e)$ liquid crystal.

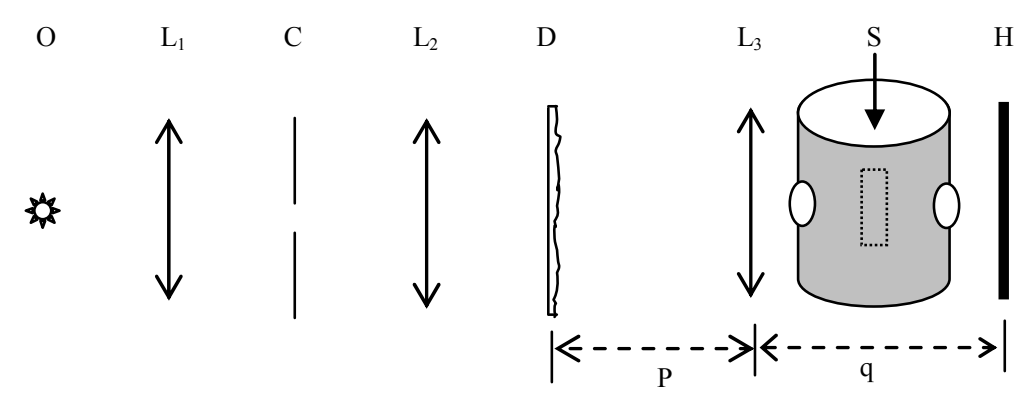

Figure 7. Optical set-up for measuring the birefringence of an anisotropic optical material. O: monochromatic source, $L_{1}, L_{2}$ \& $L_{3}$ : converging lenses, $C$ : diaphragm, D: diffuser, $S$ : liquid crystal inside the oven and $H$ : holographic plate. 
Table 2. Shows the values of birefringence $\Delta n$ at different wavelengths for the thermotropic liquid crystals used.

\begin{tabular}{ccccc}
\hline \multicolumn{5}{c}{$n$} \\
\hline Sample & $(\lambda)(632.8 \mathrm{~nm})$ & $(\lambda)(543.5 \mathrm{~nm})$ & $(\lambda)(514.5 \mathrm{~nm})$ & $(\lambda)(488 \mathrm{~nm})$ \\
\hline $6 \mathrm{e}$ & $0.21 \pm 0.01$ & $0.23 \pm 0.01$ & $0.24 \pm 0.01$ & $0.25 \pm 0.01$ \\
$8 \mathrm{e}$ & $0.23 \pm 0.01$ & $0.24 \pm 0.01$ & $0.256 \pm 0.01$ & $0.26 \pm 0.01$ \\
$14 \mathrm{e}$ & $0.27 \pm 0.01$ & $0.28 \pm 0.01$ & $0.29 \pm 0.01$ & $0.30 \pm 0.01$ \\
$6 / 14 \mathrm{e}$ & $0.20 \pm 0.01$ & $0.21 \pm 0.01$ & $0.22 \pm 0.01$ & $0.24 \pm 0.01$ \\
$6 / 14 / 16 \mathrm{e}$ & $0.16 \pm 0.01$ & $0.18 \pm 0.01$ & $0.19 \pm 0.01$ & $0.21 \pm 0.01$ \\
$6 / 8 / 14 / 16 \mathrm{e}$ & $0.14 \pm 0.01$ & $0.15 \pm 0.01$ & $0.17 \pm 0.01$ & $0.19 \pm 0.01$
\end{tabular}

where, $f$ is the focal length of the Fourier transform lens and $\lambda$ is the wavelength of light used. In order to calculate birefringence $\Delta n$, the following equation was used [15]:

$$
\frac{\Delta n}{n} \approx \frac{\lambda f}{t \sin 2 \theta}\left(\frac{p}{q \Lambda}\right)
$$

where $n$ is the refractive index of the LCs; $t$ is the thickness of the sample and $\theta$ is the angle between the optic axis and the normal to the surface of the sample. The angle $\theta$ was determined by using the conoscopic measurement system [16]. Table 2 shows the measuring data for LCs used where birefringence was calculated with laser sources of wavelengths, $632.8 \mathrm{~nm}, 543.5 \mathrm{~nm}, 514.5$ $\mathrm{nm}$, and $488 \mathrm{~nm}$, and the thickness of each sample is $(150 \mu \mathrm{m})$.

The dispersion curves for birefringence of four LCs used in the visible region are shown in Figure 8. We noted from Figure 8 that the increase of the number of carbon and hydrogen atoms in the single chemical structure, the value of birefringence as in LCs (6e), (8e) and (14e) increases. Besides, the mixture of LCs makes increase in the end group $\mathrm{NO}_{2}$ which decreases the value of birefringence as in LCs $(6 / 14 \mathrm{e}),(6 / 14 / 16 \mathrm{e})$ and $(6 / 8 / 14 /$ $16 \mathrm{e})$. These results show that in order to increase birefringence, the number of carbon and hydrogen atoms in the single chemical structure must be increased, which can be used in many applications such as liquid crystal displays. But the mixture of these liquid crystals decreases the birefringence which isn't preferable.

\section{Conclusion}

The experimental approach of speckle interferometry technique for measuring birefringence of liquid crystals and the dispersion relation was described. Also the values of refractive indices for these thermotropic liquid crystal materials were obtained with a high degree of accuracy. The experimental data shows that the values of birefringence increase by increasing the number of carbon and hydrogen atoms in the chemical structure and

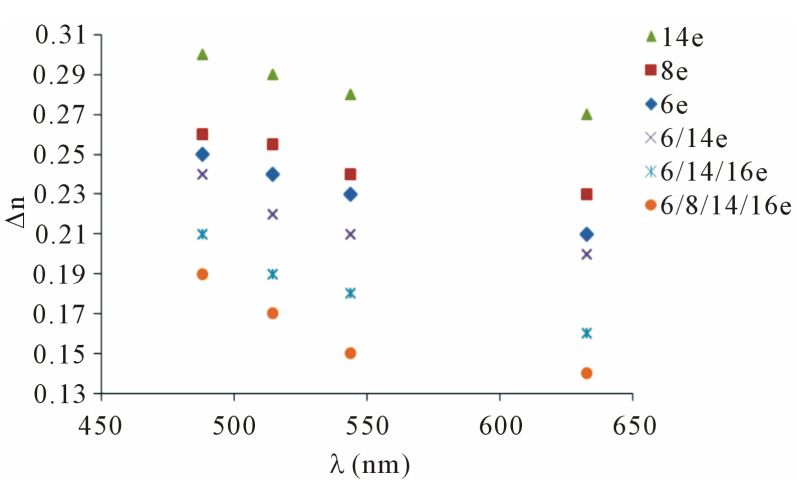

Figure 8. The dispersion relation between birefringence $(\Delta n)$ and the wavelength $\lambda$ at a certain temperature, in LC phase for $6 e, 14 e, 6 / 14 e, 6 / 14 / 16 e$ and 6/8/14/16e, liquid crystals.

decrease by increasing the end group $\mathrm{NO}_{2}$ in the mixture of liquid crystals. The end group $\mathrm{NO}_{2}$ is an electrophile which is a species having electron deficient atom, so that it is a positive charged and accepting or withdrawing electron, which decreases the stability of the chemical structure compound and then decreases the directionality of the molecules which decrease birefringence. These results become opposite when the end group is donating electrons called monotropic or nucleophiles which is a species having electrons rich atom, such as $\mathrm{OCH}_{3}$ group [17]. The experimental errors in measuring birefringence of the liquid crystal materials used were within $\pm 1 \%$ to $\pm 2 \%$, which are acceptable according to others $[15,17]$.

\section{Acknowledgements}

I wish to express my sincere thanks to Prof. Dr. T. A. ElDessouki, Faculty of Science, Ain Shams University and Prof. Dr. M. Roushdy, Physics Department, with Prof. Dr. M. Naoum, Chemistry Department, Faculty of Science, Cairo University for their facilities and guidance.

\section{REFERENCES}

[1] L. McKenna, L. S. Miller and I. R. Peterson, "Polymer Dispersed Liquid Crystal Films for Modulating Infra-Red 
Radiation," Polymer, Vol. 45, No. 20, 2004, pp. 69776984. doi:10.1016/j.polymer.2004.07.033

[2] B.-E. Benkelfata, E.-H. Horachea, Q. Zoua and B. Vinouzeb, "An Electro-Optic Modulation Technique for Direct and Accurate Measurement Of Birefringence," Optics Communication, Vol. 221, No. 4-6, 2003, pp. 271-278. doi:10.1016/S0030-4018(03)01539-6

[3] R. Manohar and J. P. Shukla, "Refractive Indices, Order Parameter and Principal Polarizability of Cholesteric Liquid Crystals and Their Homogeneous Mixtures," Journal of Physics and Chemistry of Solids, Vol. 65, No. 10, 2004, pp. 1643-1650.

[4] S. Brugioni and R. Meucci, "Mid-Infrared Interferometric Measurement of Liquid Crystal Anisotropy," Optics Communication, Vol. 230, No. 1, 2004, pp. 19-22.

[5] M. Manaia, A. Gharbia, J. P. Marceroub, H. T. Nguyenb and J. C. Rouillonb, "Optic and Electro-Optic Investigations on Smq, Smc_A and L Phases in Highly Chiral Compounds," Physica B, Vol. 368, No. 1-4, 2005, pp. 168178.

[6] F. A. Aouada , M. R. de Moura, P. R. G. Fernandes, A. F. Rubira and E. C. Muniz, "Optical and Morphological Characterization of Polyacrylamide Hydrogel and Liquid Crystal Systems," European Polymer Journal, Vol. 41, No. 9, 2005, pp. 2134-2141. doi:10.1016/j.eurpolymj.2005.03.020

[7] Z. L. Cao, L. Xuan, L. F. Hu, X. H. Lu and Q. Q. Mu, "Temperature Effect on the Diffraction Efficiency of the Liquid Crystal Spatial Light Modulator," Optics Communications, Vol. 267, No. 1, 2006, pp. 69-73. doi:10.1016/j.optcom.2006.06.003

[8] A. Jafari, A. Ghanadzadeh, H. Tajalli and M. Yeganeh, "Linear Optical Parameters of a Dyed Nematic Mixture in the Presence of an AC Voltage," Optical Materials, Vol. 29, No. 8, 2007, pp. 1004-1009. doi:10.1016/j.optmat.2006.03.034

[9] N. Bitri, A. Gharbi and J. P. Marcerou, "Scanning Conoscopy Measurement of the Optical Properties of Chiral
Smectic Liquid Crystals," Physica B: Condensed Matter, Vol. 403, No. 21-22, 2008, pp. 3921-3927. doi:10.1016/j.physb.2008.07.033

[10] Al. Bobrovsky, A. Ryabchun, A. Medvedev and V. Shibaev, "Ordering Phenomena and Photoorientation Processes in Photochromic Thin Films of LC Chiral Azobenzene-Containing Polymer Systems," Journal of Photochemistry and Photobiology A: Chemistry, Vol. 206, No. 1, 2009, pp. 46-52. doi:10.1016/j.jphotochem.2009.05.010

[11] T.-T. Tang, C.-Y. Kuo, R.-P. Pan, J.-M. Shieh and C.-L. Pan, "Strong Vertical Alignment of Liquid Crystal on Porous Anodic Aluminum Oxide Film," Journal of Display Technology, Vol. 5, No. 9, 2009, pp. 350-354.

[12] R. I. Nessim, "Phase Behaviour of Binary Mixture of 4Substituted Phenyl 4'-N-Alkoxybenzoates," Liquid Crystals, Vol. 30, No. 2, 2003, pp. 211-218. doi:10.1080/0267829021000060241

[13] E. Hecht and A. Zajac, "Optics," Addison-Wesley, Reading, Vol. 378, 1990, p. 282.

[14] M. Francon, "Information Processing Using Speckle Patterns," In: J. C. Dainty, Ed., Laser Speckle and Related Phenomena, Springer-Verlag, New York, 1975. doi:10.1007/BFb0111439

[15] L. M. Bernardo and O. D. D. Soares, "Birefringence Measurements by Double Speckle Photography," Applied Optics, Vol. 26, No. 5, 1987, pp. 769-772. doi:10.1364/AO.26.0769 1

[16] W. A. Crossland, J. H. Morrissy and B. Needham, "Tilt Angle Measurements of Nematic Phases of Cyano-Biphenyls Aligned by Obliquely Evaporated Films," Journal of Physics D: Applied Physics, Vol. 9, No. 14, 1976, p. 2001. doi:10.1088/0022-3727/9/14/010

[17] T. A. El-Dessouki, N. I. Hendawy and A. A. Zaki, "Measuring Birefringence of Curved Sheet and Single Crystals by Double-Exposure Speckle Photography," Optics and Lasers in Engineering, Vol. 47, No. 6, 2009, pp. 622-628. doi:10.1016/j.optlaseng.2008.12.006 